BESPRECHUNGEN

Anne Jenichen / Natascha Marks / Tome Sandevski (Hrsg.), Rüstungstransfers und Menschenrechte - Geschäfte mit dem Tod, Münster (LIT Verlag) 2002.

Das Buch spiegelt die Inhalte der an der Freien Universität zu Berlin gehaltenen Human Rights Lectures des Jahres 2001 wider. Ausgangspunkt aller Beiträge ist die These, dass Rüstungstransfers per se zwar nicht als Ursache von Menschenrechtsverletzung angesehen werden können, aber als derjenige Faktor, der die »Werkzeuge « (S. 7) für Menschenrechtsverletzung, z.B. Folter liefert und damit Menschenrechtsverletzer unterstützt. Rüstungstransfers werden also als die »instrumentellen Auslöser « von Menschenrechtsverletzungen bewertet (S. 9). Acht Beiträge beleuchten detailliert die Hintergründe von Rüstungstransfers und deren Konsequenzen für die Menschenrechte in vielen der Empfängerländer in folgender Reihenfolge:

Bernhard Moltmann beleuchtet zunächst den rechtlichen Rahmen für deutsche Rüstungsexporte. Sein Fazit: Ein »Zuviel« (S. 30) an rechtlichen Bestimmungen und Definitionen, sorgt für Verwirrung und die Entstehung von nutzbaren Gesetzeslücken. Die anhaltende Debatte um Rüstungsexporte in Deutschland zeige, dass die gegenwärtigen Regelungen $\mathrm{zu}$ keinem befriedigenden Ergebnis beitragen. Moltmann beklagt u.a. ein generelles Defizit an Transparenz im Kontext von rüstungsexportpolitischen Entscheidungen. Der Autor er- hofft sich eine Verbesserung der Situation durch eine Steigerung der Kohärenz in der Rüstungsexportpolitik auf EUEbene. Michael Brzoska hingegen sieht dafür in Brüssel zwar eine "wichtige", aber nicht die »entscheidende Bühne« (S. 50). Er erläutert warum für ihn eine gemeinsame Rüstungexportpolitik der EU-Mitgliedsstaaten weit entfernt liegt. Der schwere Versuch eine Harmonisierung auf europäischer Ebene $\mathrm{zu}$ erreichen, sei durch das Zögern der Mitgliedsstaaten, in diesem Bereich ihre Souveränität mit den EUGremien zu teilen, gekennzeichnet (S. 38). Brzoska zeigt dennoch die Anfänge der Entwicklung einer EU-Rüstungsexportpolitik auf. Darunter fällt z.B. der 1998 verabschiedete EU-Verhaltenskodex zur Rüstungsexportpolitik. NGOs hatten sich damals maßgeblich für eine Menschenrechtsklausel darin eingesetzt und konnten diese auch erwirken. Für Brzoska ein »merkbares Feld (S. 49) der Europäisierung der Rüstungsexportpolitik, geschmälert allerdings dadurch, dass die EU-Gremien keinerlei Kompetenzen zur Kontrolle der Einhaltung des Kodexes haben. Letztlich muss der Autor ein nüchternes Fazit ziehen: Der Lobbyismus wird auch weiterhin über Rüstungsexporte entscheiden.

Auf das Spannungsfeld zwischen wirtschaftlichen Interessen und der politischen Verantwortung gegenüber $\mathrm{zu}$ achtenden Menschenrechten geht Katja Frank ein und orientiert sich dabei an den Kontroversen des rüstungsexportpolitischen Diskurses der rot-grünen Koalition seit 1998. Schwerpunkt bildet das politische Debakel um die Entscheidung über die Lieferung eines deutschen Testpanzers an die Türkei im Herbst 1999. Um zukünftig solche politischen Debatten zu umgehen, schlägt die Autorin die Einführung eines klaren Demokratie-Kriteriums vor, das sozusagen an die Stelle des, vorher durch Moltmann beschriebenen, rechtlichen Zuviels rücken soll. Nur etablierte Demokratien sollten Empfänger von Rüstungstransfers werden dürfen, so Frank. Nicht zuletzt würde dieser Ansatz durch die allgemeine These, dass Demokratien gegeneinander nicht kriegerisch vorgehen, die Bundesrepublik oder andere Demokratien also nicht befürchten müssten selber einmal mit diesen Waffen angegriffen $\mathrm{zu}$ werden (S. $60)$, gerechtfertigt.

Peter Lock zeigt auf, dass Industrienationen unabhängig von allen Regelwerken sehr wohl Rüstungstransfers und auch vielen Konflikten in der Dritten Welt entgegenwirken könnten, wenn sie denn wollten. Konflikte würden immer häufiger durch Gewaltunternehmen geführt werden. Diese seien aber von Devisen, also Dollars abhängig, um überhaupt auf Schwarzmärkten an ihre Waffen zu kommen (S. 65). Um Devisen zu bekommen, müssen die Gewaltunternehmer sich wiederum in die internationalen Finanz- und Handelsströme einbinden und genau da böte sich die Möglichkeit für die Industrienationen das Agieren dieser Unternehmen maßgeblich zu erschweren, wenn nicht $\mathrm{zu}$ unterbinden. Lock spricht in diesem Zusammenhang sogar von einer gegenwärtigen Duldung der Schattenökonomien durch die Industrienationen, für ihn ein "politisch-moralischer Skandal« (S. 77).

Mathias John befasst sich mit Kleinwaffen als Repressionstechnologien des Alltags. Eine umfassende Definition des Begriffes »Kleinwaffe« verdeutlicht dabei, dass der Name nicht unbedingt zutreffend ist, vor allem wenn man den Schaden, den diese anrichten können, betrachtet. Im Hinblick auf geschätzte quantitative Angaben bezüglich des Umlaufs von Kleinwaffen und deren qualitativer Einsätze, spricht der Autor von einer »weltweiten Bedrohung « durch Kleinwaffen (S. 82).

Ruth Stanley widmet sich dem Thema "Kinderrechte» und Rüstungstransfers. Die völkerrechtlichen Vorgaben zu den Kinderrechten stehen im Widerspruch zur Realität des Alltags, was mit erschreckenden Zahlen belegt wird. Kinder sind die leidtragenden Opfer bewaffneter Konflikte, sie verlieren ihre Familie, ihre Existenz und werden schließlich selber »unmittelbar « missbraucht als Kindersoldaten oder Prostituierte (S. 97). Ihr Fazit: Waffentransfers müssten wahrscheinlich gänzlich eingestellt werden, wenn es einen legalen Waffenhandel ausmachte, dass er voraussehbar keine Auswirkungen auf die nahezu weltweit ratifizierten Rechte der Kinder hätte (S. 104).

Ebenfalls mit den völkerrechtlichen Aspekten von 
Rüstungstransfers setzen sich Xanthe Hall und Jens-Peter Steffen auseinander. "Das Recht auf Leben «, »das Recht auf Gesundheit « und »das Recht auf Identität « werden in Relation zu Atomtransfers und -tests gesetzt, die letztlich davon ausgehen lassen müssen, dass der Gebrauch von Atomwaffen keinesfalls auszuschließen ist. Die Autoren ziehen ein klares Fazit: die Atomenergie mit all ihren Aspekten »missachtet " generell die Menschenrechte (S. 122).

Die Konversionsentwicklung der letzten Dekade wird in dem Beitrag von Herbert Wulf erörtert. Der Autor diagnostiziert einen Stillstand »oder sogar erneute Aufrüstung « seit Ende des Kalten Krieges (S. 125). Wulf fragt aber auch nach den Erwartungen, die man an Konversion überhaupt stellen kann und welche schlicht nicht. Vor diesem Hintergrund hat für ihn durchaus eine »erfolgreiche Konversion « stattgefunden (S. 132), die aber nicht an den »überhöhten« Erwartungen am Ende des Kalten Krieges zu messen sei (S. 133). Insgesamt möchte der Autor die Abrüstungsund Konversionsbilanz seit Ende des Kalten Krieges als positiv bewerten, und macht abschließend Vorschläge $\mathrm{zu}$ einer »Revitalisierung von Abrüstung, Rüstungskontrolle und Sicherheit« (S. 139).

Ergänzend zu den oben beschriebenen Beiträgen berichten drei Autoren von der Arbeit verschiedener NGOs im Bereich der Anti-RüstungsKampagnen. Über die Geburtsstunde der Internationalen Landminenkampagne (International Campaign to ban Landmines - ICBL) und deren steinigen Weg zur Etablierung wird von Thomas Gebauer berichtet. An- hand zweier Beispiele wird das »Ausmaß des Minenproblems« verdeutlicht (S. 141). Der Autor gibt Einblicke in die Erfolgsrezepte der Organisation, die es ihr erlaubt haben, dort Beachtung zu finden, wo es am Anfang der Kampagne von vielen für »unmöglich gehalten « wurde, namentlich der Militärund Sicherheitspolitik (S. 147). Während der Autor beschreibt, was bisher erreicht werden konnte, klärt er auch über die bestehenden Defizite auf, z.B. dass die wichtigsten minenexportierenden Länder dem Abkommen bisher nicht beigetreten sind.

Andrea Kolling schreibt über die Kampagne "Stoppt den Rüstungsexport « des »BUKO«, dem »Bundeskongress entwicklungspolitischer Aktionsgruppen «, der als ein Dachverband verschiedener Aktionsgruppen agiert. Die Autorin berichtet über die bisherigen »Highlights « der Kampagne (S. 155) und zeigt auf, wie das Ende des Kalten Krieges auch hier die Bedingungen geändert hat und Anti-Rüstungs-Kampagnen eher schwieriger gemacht hat, als einfacher. Speziell in Deutschland habe sich im Themenbereich vieles für die engagierten NGOs mit dem Regierungswechsel 1998 verändert: Was »heißt das, wenn die ehemaligen Bündnispartner auf der Regierungsbank sitzen?«(S. 158).

Ein chronologischer Überblick über das Programm von Amnesty International (AI), Rüstungstransfers $\mathrm{zu}$ stoppen, wird von Matthias John geboten. Während die Organisation zunächst gegen Rüstungstransfers von Regierungen mobilisierte, hatte $\mathrm{AI}$ auch schon früh den Transfers von Kleinwaffen Aufmerksamkeit geschenkt. Seit Mitte der 90er Jahre bildet die »Offenlegung des Schattenmarktes der Folter- und Zwangswerkzeuge « den Arbeitsschwerpunkt (S. 165).

Insgesamt bieten die Beiträge einen thematischen Überblick, dem gut zu folgen ist. Alle Beiträge sind durch Definitionen und quantitative Angaben ergänzt. Der Band zeichnet sich besonders durch die Vielfältigkeit der Beiträge und ihrer »Quintessenzen« aus. Ein Querschnitt wissenschaftlicher Einschätzungen teils nüchtern, teils sehr idealistisch ist das Ergebnis. Die Beiträge über die Arbeit und Erfahrungen dreier NGOs spiegeln letztlich alle dieser Einschätzung en wider. Sie zeigen, dass sich mit Idealismus und Engagement im Themenfeld der Rüstungstransfers etwas bewegen lässt, aber dass die Ergebnisse stets auch nüchterne, real-politische Facetten in sich tragen, und das an entscheidender Stelle. Alle Autoren sind Mitarbeiterinnen und Mitarbeiter verschiedener Nichtregierungsorganisationen und wissenschaftlicher Institutionen. Ein umfassendes, kommentiertes Adressenverzeichnis mit Forschungsinstitutionen und NGOs rundet den Band ab und regt den Leser zum Engagement an.

\section{Vivien-Marie Drews}

\section{Kai Ambos/Jörg Arnold}

(Hrsg.), Der Irak-Krieg und das Völkerrecht. Juristische Zeitgeschichte, Abteilung 5: Juristisches Zeitgeschehen Rechtspolitik und Justiz aus zeitgenössischer Perspektive, Band 14, Berlin (Berliner Wissenschafts-Verlag) 2004.

Täglich stellen mehr und mehr Belege die offiziellen Begründungen der USA für den Irak-Krieg in Frage, erschüttern Meldungen von neuen Terrorakten in iraki- schen Städten die Weltöffentlichkeit, wird es immer offenkundiger, daß der schnelle Sieg der Alliierten weder Sicherheit für das irakische Volk noch für die internationale Gemeinschaft gebracht hat. In der Folge tendiert die aktuelle Diskussion um den Irak-Krieg zu einer verantwortungsethischen Debatte, die dessen Rechtfertigung in der Effektivität der Zielerreichung $\mathrm{zu}$ suchen scheint, wohingegen dessen Vereinbarkeit mit den kodifizierten Werten unserer und der Weltgesellschaft aus dem Fokus des Interesses gerückt ist.

Gegen diesen Trend wollen die Herausgeber von »Der Irak-Krieg und das Völkerrecht « - beide Referenten bzw. ehemalige Referenten am Max-Planck-Institut für ausländisches und internationales Strafrecht in Freiburg im Breisgau - mit ihrem Sammelband ein juristisches Zeugnis ablegen, das den wissenschaftlichen Diskurs um das Verhältnis von Recht und Politik neu beleben soll. Schließlich ging das Völkerrecht aus der bis dato einzigartigen Auseinandersetzung um einen internationalen Konflikt in der Öffentlichkeit und im UN-Sicherheitsrat als Verlierer hervor. Rechtliche Argumente spielten nach Ansicht der Herausgeber in der Irak-Frage kaum eine Rolle: Zum einen brachen die »Falken « angesichts des unüberwindbaren Widerstands der »Tauben« das Völkerrecht, indem sie die Zuständigkeit des UN-Sicherheitsrates ignorierten. Zum anderen traten nicht einmal alle Kriegsgegner selbst explizit für die Stärkung des internationalen Rechts ein. Auf beiden Seiten dominierten stattdessen politische oder wirtschaftliche Argumente. Aus diesen Gründen wollen Ambos und Ar- 
nold mit ihrer Anthologie zum Thema Irak-Krieg und Völkerrecht dazu beitragen, »dem Völkerrecht wieder den ihm gebührenden Rang als Ordnungs- und Machtbegrenzungsrecht zuzuweisen « (S. XV). Zwar geht es ihnen darum, rechtliche Einwände gegen eine Rückkehr des Faustrechts in die Internationalen Beziehungen vorzubringen, nichtsdestotrotz lassen sie durchaus Vertreter gegensätzlicher juristischer Positionen zu Wort kommen.

Das Spektrum der Quellen, aus denen die insgesamt 78 Beiträge von ca. Juli 2002 bis Mai 2003 ausgewählt wurden, erstreckt sich in erster Linie auf die überregionale deutschsprachige Presse. Die meisten Artikel, Interviews und Kommentare stammen aus Tageszeitungen aller politischen Couleur, darunter die Neue Zürcher Zeitung, Frankfurter Allgemeine Zeitung, Financial Times Deutschland, Tagesspiegel, Süddeutsche Zeitung, Berliner Zeitung, Frankfurter Rundschau, die tageszeitung sowie Junge Welt. Berücksichtigt wurden außerdem Die Zeit, Spiegel und Focus, ai-Journal sowie einige juristische Fachzeitschriften, desweiteren Stellungnahmen und Ausarbeitungen von Juristenverbänden wie IALANA und VDJ, der Deutschen Gesellschaft für die Vereinten Nationen sowie von Forschungseinrichtungen wie den Wissenschaftlichen Diensten des Deutschen Bundestages, dem Institut für Friedensforschung und Sicherheitspolitik an der Universität Hamburg und der Berlin-Brandenburgischen Akademie der Wissenschaften. Auch die Pressemitteilung des Generalbundesanwalts beim Bundesgerichtshof zur Frage des Straftatbestands der Vorbereitung eines Angriffskrieges sowie die sog. "AWACSEntscheidung « des Bundesverfassungsgerichts nebst Urteilsbegründung sind in Gänze abgedruckt. Auf Beiträge aus internationalen Quellen wurde mit Ausnahme von Le Monde Diplomatique verzichtet.

Gegliedert ist die 530 Seiten zählende Dokumentation in drei Bereiche: Im ersten Teil stehen rechtsphilosophische, rechtspolitische, rechtsge- schichtliche sowie sozialwissenschaftliche Aspekte im Vordergrund, die ein Vorverständnis für die Problematik vermitteln sollen. Die Anordnung der Beiträge erfolgt chronologisch nach Erscheinungsdaten. Angesprochen werden Themen wie der gerechte Krieg, die neue Weltordnung und Transformation der Diktatur. Der zweite Teil widmet sich Fragestellungen, welche sich vorrangig aus dem deutschen Recht ergeben. Dies betrifft insbesondere das Verbot des Angriffskriegs und dessen Unterstützung, damit zusammenhängend die Gewährung von Überflugrechten und den Einsatz deutscher Soldaten in AWACS-Flugzeugen sowie das Spannungsverhältnis von NATOBündnisverpflichtungen und Verfassungs- und Völkerrecht. Der dritte und weitaus größte Teil untersucht die völkerrechtliche Zulässigkeit des Krieges gegen den Irak im Allgemeinen wie unter besonderer Berücksichtigung der Rolle von UN-Sicherheitsrat und Internationalem Gerichtshof, von Humanitärem Völkerrecht und Völkerstrafrecht: Wie weit reichten die
Resolutionen des UN-Sicherheitsrates? Begingen die USA Völkerrechtsbruch? Können Präventivkriege rechtmäßig sein? Was bedeutet der Ausgang des Konflikts für das System der UN und die Rolle des Völkerrechts? Diesen und ähnlichen Fragen wird nachgegangen. Im Anhang schließlich finden sich die einschlägigen Resolutionen des UNSicherheitsrates im englischen Original und in der offiziellen deutschen Übersetzung.

Autoren der gesammelten Beiträge sind Journalisten, Wissenschaftler und Praktiker, in erster Linie natürlich namhafte Völker- und Staatsrechtler. Als Adressat kommt neben dem Fachpublikum zweifellos die breite Öffentlichkeit in Frage, handelte es sich beim Irak-Krieg doch um einen Konflikt, der in ungeahnter Weise zu einer Mobilisierung von großen Teilen der Gesellschaft, zu nationalen wie internationalen politischen Kontroversen geführt hat.

Bertram Kühnreich 